\title{
A Class of Special Hypersurfaces in Real Space Forms
}

\author{
Yan Zhao ${ }^{1,2}$ and Ximin Liu ${ }^{1}$ \\ ${ }^{1}$ School of Mathematical Sciences, Dalian University of Technology, Dalian 116024, China
}

${ }^{2}$ Henan Light Industry School, Zhengzhou 450000, China

Correspondence should be addressed to Yan Zhao; zy4012006@126.com

Received 15 January 2016; Revised 18 May 2016; Accepted 26 May 2016

Academic Editor: Hugo Leiva

Copyright (c) 2016 Y. Zhao and X. Liu. This is an open access article distributed under the Creative Commons Attribution License, which permits unrestricted use, distribution, and reproduction in any medium, provided the original work is properly cited.

We define the generalized golden- and product-shaped hypersurfaces in real space forms. A hypersurface $M$ in real space forms $R^{n+1}$, $S^{n+1}$, and $H^{n+1}$ is isoparametric if it has constant principal curvatures. Based on the classification of isoparametric hypersurfaces, we obtain the whole families of the generalized golden- and product-shaped hypersurfaces in real space forms.

\section{Introduction}

The golden ratio, which sometimes is called golden number, golden section, golden proportion, or golden mean, has many applications in many parts of mathematics, natural sciences, music, art, philosophies, and computational science [1]. In the past few years, the golden ratio has played a more and more significant role in modern physical research and atomic physics [2]. The golden ratio also has interesting properties in topology of four-manifolds, in conformal field theory, in mathematical probability theory, in Cantorian spacetime [3], and in differential geometry.

The notion of golden structure on a manifold $M$ was introduced in $[4,5]$ as a $(1,1)$-tensor field on $M$ which satisfies the equation

$$
J^{2}=J+I
$$

where $I$ is the usual Kronecker tensor field of $M$. It attracts many authors' attentions to focus on a class of well-known objects, namely, hypersurfaces in real space forms. It is interesting to notice that they are hyperspheres, a hyperbolic hyperplane in the hyperbolic case, or a generalized Clifford torus in the spherical space form, and this can be a new motivation to see the sphere as a golden-shaped surface. A well-known result of parallelism for the shape operators satisfying a quadratic equation is applied also for the spherical framework. More precisely, all golden (as well as product) hypersurfaces in real space forms are parallel hypersurfaces.
Crasmareanu and Hrețcanu derived a natural correspondence between golden structures and almost product structures in [4]. Recently, the golden- and product-shaped hypersurfaces in real space forms were defined and the whole families of golden- and product-shaped hypersurfaces were obtained in [6]. Similar to the golden structure on a manifold $M$, in [7], Crasmareanu and Hreţcanu defined the metallic structure on a manifold $M$. The metallic structure on a manifold $M$ is a $(1,1)$-tensor field on $M$ satisfying the equation

$$
J^{2}=p J+q I,
$$

where $I$ is the usual Kronecker tensor field of $M$ and $p, q$ are positive integers [7]. In the present study, the notion of a metallic shaped hypersurface was defined and the full classification of metallic shaped hypersurfaces in real space forms was obtained in [8].

In this paper, we define the generalized golden- and product-shaped hypersurfaces in real space forms. Based on the classification of isoparametric hypersurfaces, we obtain the whole families of the generalized golden- and productshaped hypersurfaces in real space forms.

\section{Generalized Golden-Shaped Hypersurfaces}

Let $M$ be an oriented embedded hypersurface of the real space form $M^{n+1}(c)$ and, for a certain normal field $N$, let $A=A_{N}$ be the associated shaped operator (see [9]); then 
$\mu_{1}, \ldots, \mu_{n}$ will be the principal curvatures of $M$ (see [10] for the semi-Euclidean case). We will take $c \in\{-1,0,1\}$ and consequently $M^{n+1}(0)=\mathbb{R}^{n+1}, M^{n+1}(1)=\mathbb{S}^{n+1}=S^{n+1}(1)$, respectively, $M^{n+1}(-1)=\mathbb{U}^{n+1}$.

Definition 1. $M$ is called a golden-shaped hypersurface if $A$ is a golden structure; that is, $A^{2}=A+I$, where $I$ is the identity on the tangent bundle of $M$.

Definition 2. $M$ is called a metallic shaped hypersurface if the shaped operator $A$ is a metallic structure.

In this paper, we give the definition of generalized goldenshaped hypersurfaces.

Definition 3. $M$ is called a generalized golden-shaped hypersurface if $A$ is a generalized golden structure; that is, $A^{2}=$ $a A+b I$, where $I$ is the identity on the tangent bundle of $M$ and $a, b$ are real constants satisfying $a^{2}+4 b \geq 0$.

Cartan's Identity. Let $f: M \rightarrow M^{n+1}(c)$ be an isoparametric hypersurface with $g$ distinct principal curvatures $\mu_{1}, \ldots, \mu_{g}$, having respective multiplicities $m_{1}, \ldots, m_{g}$. If $g>1$, for each $i, 1 \leq i \leq g$,

$$
\sum_{j \neq i} m_{j} \frac{c+\mu_{i} \mu_{j}}{\mu_{i}-\mu_{j}}=0 .
$$

The important equation, known as Cartan's identity, is crucial in Cartan's work on isoparametric hypersurfaces.

In the case $c=0$, there are at most two distinct principal curvatures and if there are two, then one of them must be zero.

In the case $c=-1$, there are at most two distinct principal curvatures and if there are two, then they are reciprocals of each other.

In the case $c=1$ and if there are two distinct principal curvatures, then they are negative reciprocals of each other.

Suppose $M$ is a generalized golden-shaped hypersurface. It follows that the principal curvatures of $M$, as eigenvalues of $A$, are $\lambda_{1}=\left(a+\sqrt{a^{2}+4 b}\right) / 2$ and $\lambda_{2}=\left(a-\sqrt{a^{2}+4 b}\right) / 2$ if $a^{2}+4 b>0$. The principal curvatures of $M$, as eigenvalues of $A$, especially, are $\lambda_{1}=\lambda_{2}=a / 2$ if $a^{2}+4 b=0$. According to [11], the manifold $M$ is an isoparametric hypersurface and this yields the following theorems.

Theorem 4. The only generalized golden-shaped hypersurfaces of $\mathbb{R}^{n+1}$ are as follows.

Case $1\left(\right.$ If $\left.a^{2}+4 b=0\right)$.

(1) If $a \neq 0$,

$$
M=S^{n}\left(\frac{2}{|a|}\right) .
$$

(2) If $a=0$,

$$
M=\left\{x \mid x^{n+1}=0\right\}
$$

which is totally geodesic and is isometric to $\mathbb{R}^{n}$. In this case, the second fundamental form $A$ is zero.

Case 2 (If $\left.a^{2}+4 b>0\right)$.

(1) If $b \neq 0$,

$$
\begin{aligned}
& M=S^{n}\left(\frac{1}{\left|\lambda_{1}\right|}\right), \\
& M=S^{n}\left(\frac{1}{\left|\lambda_{2}\right|}\right) .
\end{aligned}
$$

(2) If $b=0$, there are the following three cases:

(i) $M=\left\{x \mid x^{n+1}=0\right\}$; in this case, the second fundamental form $A$ is zero; $M$ is totally geodesic and is isometric to $\mathbb{R}^{n}$;

(ii) $M=S^{n}(1 /|a|)$;

(iii) for $1<k<n$,

$$
M=\left\{x \mid\left(x^{1}\right)^{2}+\cdots+\left(x^{k}\right)^{2}=\frac{1}{a^{2}}\right\}=S^{k} \times R^{n-k}
$$

$$
\text { with } A=a I_{k} \oplus O \text {. }
$$

Proof.

Case 1. Suppose $a^{2}+4 b=0$ and $\lambda_{1}=\lambda_{2}=a / 2$; we distinguish the following two cases.

(1) If $a \neq 0$ and $\lambda_{1}=\lambda_{2} \neq 0$, we get $\mu_{1}=\mu_{2}=\cdots=$ $\mu_{n}=a / 2$ and $c=a^{2} / 4>0$; then $M$ is totally umbilical and $M=S^{n}(2 /|a|)$.

(2) If $a=0$ and $\lambda_{1}=\lambda_{2}=0$, we get $\mu_{1}=\mu_{2}=\cdots=\mu_{n}=0$ and $c=0$; then $M$ is totally umbilical and $M=\left\{x \mid x^{n+1}=0\right\}$ which is totally geodesic and is isometric to $\mathbb{R}^{n}$.

Case 2. Suppose $a^{2}+4 b \neq 0$; we distinguish the following cases.

(1) If $b \neq 0$ and $\mu_{1}=\mu_{2}=\cdots=\mu_{n}=\lambda_{1} \neq 0$, we get $c=\lambda_{1}^{2}>0$. Then, $M$ is totally umbilical and $M=S^{n}\left(1 /\left|\lambda_{1}\right|\right)$ which presents the first above sphere.

If $b \neq 0$ and $\mu_{1}=\mu_{2}=\cdots=\mu_{n}=\lambda_{2} \neq 0$, we get $c=\lambda_{2}^{2}>$ 0 . Then, $M$ is totally umbilical too and $M=S^{n}\left(1 /\left|\lambda_{2}\right|\right)$ which presents the second above sphere.

(2) If $b=0$, then $a \neq 0$ and $\lambda_{1}=0, \lambda_{2}=a$, or $\lambda_{1}=a$, $\lambda_{2}=0$.

(i) If $\mu_{1}=\mu_{2}=\cdots=\mu_{n}=0$, we get $c=0$. Then $M$ is totally umbilical and $M=\left\{x \mid x^{n+1}=0\right\}$. In this case, the second fundamental form $A$ is zero; $M$ is totally geodesic and is isometric to $\mathbb{R}^{n}$.

(ii) If $\mu_{1}=\mu_{2}=\cdots=\mu_{n}=a \neq 0$, we get $c=a^{2}>0$. Then $M$ is totally umbilical and $M=S^{n}(1 /|a|)$.

(iii) For $1<k<n$, if $\mu_{1}=\cdots=\mu_{k}=a \neq 0$ and $\mu_{k+1}=$ $\cdots=\mu_{n}=0$, then $c_{1}=a^{2}>0$ and $c_{2}=0$. We get $M=\left\{x \mid\left(x^{1}\right)^{2}+\cdots+\left(x^{k}\right)^{2}=1 / a^{2}\right\}=S^{k} \times R^{n-k}$ with $A=a I_{k} \oplus O$. 
Recall that the hyperbolic space in the upper half-space model is defined as

$$
\begin{aligned}
& \mathbb{H}^{n+1}=\left\{x \in \mathbb{R}^{n+2}:\left(x^{1}\right)^{2}+\cdots+\left(x^{n+1}\right)^{2}-\left(x^{n+2}\right)^{2}\right. \\
& \left.=-1, x^{n+2}>0\right\}
\end{aligned}
$$

and the isoparametric hypersurfaces of it are [12, P. 252].

(i) $M_{h, 1, k}=\left\{x \in \mathbb{H}^{n+1} ; x^{k}=0\right\}$, for $1 \leq k \leq n+1$ with $A=O ; M$ is totally geodesic and is in fact just $\mathbb{T}^{n}$.

(ii) $M_{h, 2, k, r}=\left\{x \in \mathbb{H}^{n+1} ; x^{k}=r>0\right\}$, for $1 \leq k \leq$ $n+1$ with $A=\sqrt{c+1} I$, where $c=-1 / r^{2} \in(-1,0)$; then $r \in(1,+\infty)$; in this case $M$ is isometric to $\mathbb{H}^{n}(c)$.

(iii) $M_{h, 3}=\left\{x \in \mathbb{T}^{n+1} ; x^{n+2}=x^{n+1}+1\right\}$ with $A=I$; $M$ is isometric to $\mathbb{R}^{n}$.

(iv) $M_{h, 4, r}=\left\{x \in \mathbb{H}^{n+1} ;\left(x^{1}\right)^{2}+\cdots+\left(x^{n+1}\right)^{2}=r^{2}\right\}=$ $\mathbb{H}^{n+1} \cap S^{n}(r)$ with $A=\sqrt{c+1} I$, where $c=1 / r^{2}>0$; in this case $M$ is isometric to $S^{n}(r)$.

(v) $M_{h, 5, k, r}=\left\{x \in \mathbb{T}^{n+1} ;\left(x^{1}\right)^{2}+\cdots+\left(x^{k+1}\right)^{2}=r^{2}\right\}=$ $\mathbb{\boxplus}^{n+1} \cap S^{k}$, for $1 \leq k \leq n$ with $A=\lambda I_{k} \oplus(1 / \lambda) I_{n-k}$, where $\lambda=\sqrt{r^{2}+1} / r>0$.

Theorem 5. The only generalized golden-shaped hypersurfaces of $\mathbb{M}^{n+1}$ are as follows.

Case $1\left(\right.$ If $\left.a^{2}+4 b=0\right)$.

(i) If $a=0$, for $1 \leq k \leq n+1$,

$$
M_{h, 1, k}=\left\{x \in \mathbb{T}^{n+1} ; x^{k}=0\right\} .
$$

In this case $A=O ; M$ is totally geodesic and is in fact just $\mathbb{H}^{n}$.

(ii) If $a \in(-2,0) \cup(0,2)$, for $1 \leq k \leq n+1$,

$$
M_{h, 2, k, 2 / \sqrt{4-a^{2}}}=\left\{x \in \mathbb{M}^{n+1} ; x^{k}=\frac{2}{\sqrt{4-a^{2}}}\right\}
$$

which is isometric to $\mathbb{H}^{n}\left(a^{2} / 4-1\right)$.

(iii) If $a= \pm 2$,

$$
M_{h, 3}=\left\{x \in \mathbb{M}^{n+1} ; x^{n+2}=x^{n+1}+1\right\}
$$

which is isometric to $\mathbb{R}^{n}$.

(iv) If $a \in(-\infty,-2) \cup(2,+\infty)$,

$$
\begin{aligned}
& M_{h, 4,2 / \sqrt{a^{2}-4}} \\
& \quad=\left\{x \in \mathbb{H}^{n+1} ;\left(x^{1}\right)^{2}+\cdots+\left(x^{n+1}\right)^{2}=\frac{4}{a^{2}-4}\right\}
\end{aligned}
$$

which is isometric to $S^{n}\left(2 / \sqrt{a^{2}-4}\right)$.

Case 2 (If $\left.a^{2}+4 b>0\right)$.

(i) Suppose $\mu_{1}=\mu_{2}=\cdots=\mu_{n}=\lambda_{1}$; there are the following four cases.

(1) If $(a, b) \in\{(a, b) \mid b=0, a<0\}$, for $1 \leq k \leq n+1$,

$$
M_{h, 1, k}=\left\{x \in \mathbb{H}^{n+1} ; x^{k}=0\right\} .
$$

In this case $A=O ; M$ is totally geodesic and is in fact just $\mathbb{H}^{n}$.

(2) If $(a, b) \in\{(a, b) \mid a+b-1<0, a<2\} \backslash\{(a, b) \mid$ $a<0, b=0\} \backslash\{(a, b) \mid a-b+1 \geq 0, a \leq-2\}$, for $1 \leq k \leq n+1$,

$$
M_{h, 2, k, 1 / \sqrt{1-\lambda_{1}^{2}}}=\left\{x \in \mathbb{H}^{n+1} ; x^{k}=\frac{1}{\sqrt{1-\lambda_{1}^{2}}}\right\}
$$

which is isometric to $\mathbb{M}^{n}\left(\lambda_{1}^{2}-1\right)$.

(3) If $(a, b) \in\{(a, b) \mid a-b+1=0, a<-2\} \cup\{(a, b) \mid$ $a+b-1=0, a<2\}$,

$$
M_{h, 3}=\left\{x \in \mathbb{T}^{n+1} ; x^{n+2}=x^{n+1}+1\right\},
$$

which is isometric to $\mathbb{R}^{n}$.

(4) If $(a, b) \in\{(a, b) \mid a+b-1>0, a<2\} \cup\{(a, b) \mid a \geq$ $2\} \cup\{(a, b) \mid a<-2, a-b+1>0\}$. we get $\lambda_{1}^{2}>1$ and $c=\lambda_{1}^{2}-1>0$ :

$$
\begin{aligned}
& M_{h, 4,1 / \sqrt{\lambda_{1}^{2}-1}} \\
& \quad=\left\{x \in \mathbb{M}^{n+1} ;\left(x^{1}\right)^{2}+\cdots+\left(x^{n+1}\right)^{2}=\frac{1}{\lambda_{1}^{2}-1}\right\},
\end{aligned}
$$

which is isometric to $S^{n}\left(1 / \sqrt{\lambda_{1}^{2}-1}\right)$.

(ii) Suppose $\mu_{1}=\mu_{2}=\cdots=\mu_{n}=\lambda_{2}$; there are the following four cases.

(1) If $(a, b) \in\{(a, b) \mid b=0, a>0\}$, for $1 \leq k \leq n+1$,

$$
M_{h, 1, k}=\left\{x \in \mathbb{H}^{n+1} ; x^{k}=0\right\} .
$$

In this case $A=O ; M$ is totally geodesic and is in fact just $\mathbb{U}^{n}$.

(2) If $(a, b) \in\{(a, b) \mid a-b+1>0, a>-2\} \backslash\{(a, b) \mid$ $a>0, b=0\} \backslash\{(a, b) \mid a \geq 2, a+b-1 \leq 0\}$, for $1 \leq k \leq n+1$,

$$
M_{h, 2, k, 1 / \sqrt{1-\lambda_{2}^{2}}}=\left\{x \in \mathbb{M}^{n+1} ; x^{k}=\frac{1}{\sqrt{1-\lambda_{2}^{2}}}\right\}
$$

which is isometric to $\mathbb{\boxplus}^{n}\left(\lambda_{2}^{2}-1\right)$.

(3) If $(a, b) \in\{(a, b) \mid a-b+1=0, a>-2\} \cup\{(a, b) \mid$ $a+b-1=0, a>2\}$,

$$
M_{h, 3}=\left\{x \in \mathbb{H}^{n+1} ; x^{n+2}=x^{n+1}+1\right\}
$$

which is isometric to $\mathbb{R}^{n}$. 
(4) If $(a, b) \in\{(a, b) \mid a-b+1<0, a>-2\} \cup\{(a, b) \mid$ $a \leq-2\} \cup\{(a, b) \mid a>2, a+b-1<0\}$,

$$
\begin{aligned}
& M_{h, 4,1 / \sqrt{\lambda_{2}^{2}-1}} \\
& \quad=\left\{x \in \mathbb{H}^{n+1} ;\left(x^{1}\right)^{2}+\cdots+\left(x^{n+1}\right)^{2}=\frac{1}{\lambda_{2}^{2}-1}\right\}
\end{aligned}
$$

which is isometric to $S^{n}\left(1 / \sqrt{\lambda_{2}^{2}-1}\right)$.

(iii) Suppose $\mu_{1}=\mu_{2}=\cdots=\mu_{k}=\lambda_{1}$, and $\mu_{k+1}=\cdots=$ $\mu_{n}=\lambda_{2}$, for $1 \leq k \leq n$.

If $b=-1$,

$$
\begin{aligned}
& M_{h, 5, k, 1 / \sqrt{\lambda_{1}^{2}-1}} \\
& \quad=\left\{x \in \mathbb{T}^{n+1} ;\left(x^{1}\right)^{2}+\cdots+\left(x^{k+1}\right)^{2}=\frac{1}{\lambda_{1}^{2}-1}\right\}
\end{aligned}
$$

with $A=\lambda_{1} I_{k} \oplus\left(1 / \lambda_{1}\right) I_{n-k}$.

Proof.

Case 1. Suppose $a^{2}+4 b=0, \lambda_{1}=\lambda_{2}=a / 2$; we distinguish the following four cases.

(i) If $a=0$, we get $\mu_{1}=\mu_{2}=\cdots=\mu_{n}=a / 2=0$ and $c=-1$. Then for $1 \leq k \leq n+1$

$$
M_{h, 1, k}=\left\{x \in \mathbb{T}^{n+1} ; x^{k}=0\right\} .
$$

In this case $A=O ; M$ is totally geodesic and is in fact just $\mathbb{H}^{n}$.

(ii) If $a \in(-2,0) \cup(0,2)$, we get $\mu_{1}=\mu_{2}=\cdots=\mu_{n}=a / 2$ and $-1<c=a^{2} / 4-1<0$. Then, for $1 \leq k \leq n+1$, $M_{h, 2, k, 2 / \sqrt{4-a^{2}}}=\left\{x \in \mathbb{H}^{n+1} ; x^{k}=2 / \sqrt{4-a^{2}}\right\}$ which is isometric to $\mathbb{H}^{n}\left(a^{2} / 4-1\right)$.

(iii) If $a= \pm 2$, we get $\mu_{1}=\mu_{2}=\cdots=\mu_{n}= \pm 1$ and $c=0$. Then $M_{h, 3}=\left\{x \in \mathbb{H}^{n+1} ; x^{n+2}=x^{n+1}+1\right\}$ with $A=I$ which is isometric to $\mathbb{R}^{n}$.

(iv) If $a \in(-\infty,-2) \cup(2,+\infty)$, we get $\mu_{1}=\mu_{2}=\cdots=$ $\mu_{n}=a / 2$ and $c=a^{2} / 4-1>0$. Then $M_{h, 4,2 / \sqrt{a^{2}-4}}=\{x \in$ $\left.\mathbb{H}^{n+1} ;\left(x^{1}\right)^{2}+\cdots+\left(x^{n+1}\right)^{2}=4 /\left(a^{2}-4\right)\right\}$ which is isometric to $S^{n}\left(2 / \sqrt{a^{2}-4}\right)$.

Case 2 (If $a^{2}+4 b>0$ ).

(i) Suppose $\mu_{1}=\mu_{2}=\cdots=\mu_{n}=\lambda_{1}$; there are the following four cases.

(1) If $(a, b) \in\{(a, b) \mid b=0, a<0\}$, we get $\lambda_{1}=(a+$ $\left.\sqrt{a^{2}+4 b}\right) / 2=(a+|a|) / 2=0$. That is to say, $\mu_{1}=$ $\mu_{2}=\cdots=\mu_{n}=0$ and $c=-1$. Then, for $1 \leq k \leq n+1$, $M_{h, 1, k}=\left\{x \in \mathbb{H}^{n+1} ; x^{k}=0\right\}$. In this case $A=O ; M$ is totally geodesic and is in fact just $\mathbb{M}^{n}$.

(2) If $(a, b) \in\{(a, b) \mid a+b-1<0, a<2\} \backslash\{(a, b) \mid a<$ $0, b=0\} \backslash\{(a, b) \mid a-b+1 \geq 0, a \leq-2\}$, we get $0<\lambda_{1}^{2}<1$ and $-1<c=\lambda_{1}^{2}-1<0$. Then, for $1 \leq k \leq$ $n+1, M_{h, 2, k, 1 / \sqrt{1-\lambda_{1}^{2}}}=\left\{x \in \mathbb{M}^{n+1} ; x^{k}=1 / \sqrt{1-\lambda_{1}^{2}}\right\}$, which is isometric to $\mathbb{H}^{n}\left(\lambda_{1}^{2}-1\right)$.
(3) If $(a, b) \in\{(a, b) \mid a-b+1=0, a<-2\} \cup\{(a, b) \mid$ $a+b-1=0, a<2\}$, we get $\lambda_{1}= \pm 1$ and $c=0$. Then $M_{h, 3}=\left\{x \in \mathbb{H}^{n+1} ; x^{n+2}=x^{n+1}+1\right\}$, which is isometric to $\mathbb{R}^{n}$.

(4) If $(a, b) \in\{(a, b) \mid a+b-1>0, a<2\} \cup\{(a, b) \mid$ $a \geq 2\} \cup\{(a, b) \mid a<-2, a-b+1>0\}$, we get $\lambda_{1}^{2}>1$ and $c=\lambda_{1}^{2}-1>0$. Then $M_{h, 4,1 / \sqrt{\lambda_{1}^{2}-1}}=\{x \in$ $\left.\mathbb{H}^{n+1} ;\left(x^{1}\right)^{2}+\cdots+\left(x^{n+1}\right)^{2}=1 /\left(\lambda_{1}^{2}-1\right)\right\}$, which is isometric to $S^{n}\left(1 / \sqrt{\lambda_{1}^{2}-1}\right)$.

(ii) Suppose $\mu_{1}=\mu_{2}=\cdots=\mu_{n}=\lambda_{2}$; there are the following four cases.

(1) If $(a, b) \in\{(a, b) \mid b=0, a>0\}$, we get $\lambda_{2}=(a-$ $\left.\sqrt{a^{2}+4 b}\right) / 2=(a-|a|) / 2=0$. That is to say, $\mu_{1}=$ $\mu_{2}=\cdots=\mu_{n}=0$ and $c=-1$. Then, for $1 \leq k \leq n+1$, $M_{h, 1, k}=\left\{x \in \mathbb{H}^{n+1} ; x^{k}=0\right\}$. In this case $A=O ; M$ is totally geodesic and is in fact just $\mathbb{H}^{n}$.

(2) If $(a, b) \in\{(a, b) \mid a-b+1>0, a>-2\} \backslash\{(a, b) \mid$ $a>0, b=0\} \backslash\{(a, b) \mid a \geq 2, a+b-1 \leq 0\}$, we get $0<\lambda_{2}^{2}<1$ and $-1<c=\lambda_{2}^{2}-1<0$. Then, for $1 \leq k \leq$ $n+1, M_{h, 2, k, 1 / \sqrt{1-\lambda_{2}^{2}}}=\left\{x \in \mathbb{H}^{n+1} ; x^{k}=1 / \sqrt{1-\lambda_{2}^{2}}\right\}$, which is isometric to $\mathbb{H}^{n}\left(\lambda_{2}^{2}-1\right)$.

(3) If $(a, b) \in\{(a, b) \mid a-b+1=0, a>-2\} \cup\{(a, b) \mid$ $a+b-1=0, a>2\}$, we get $\lambda_{2}= \pm 1$ and $c=0$. Then $M_{h, 3}=\left\{x \in \mathbb{H}^{n+1} ; x^{n+2}=x^{n+1}+1\right\}$, which is isometric to $\mathbb{R}^{n}$.

(4) If $(a, b) \in\{(a, b) \mid a-b+1<0, a>-2\} \cup\{(a, b) \mid$ $a \leq-2\} \cup\{(a, b) \mid a>2, a+b-1<0\}$, we get $\lambda_{2}^{2}>1$ and $c=\lambda_{2}^{2}-1>0$. Then $M_{h, 4,1 / \sqrt{\lambda_{2}^{2}-1}}=\{x \in$ $\left.\mathbb{H}^{n+1} ;\left(x^{1}\right)^{2}+\cdots+\left(x^{n+1}\right)^{2}=1 /\left(\lambda_{2}^{2}-1\right)\right\}$, which is isometric to $S^{n}\left(1 / \sqrt{\lambda_{2}^{2}-1}\right)$.

(iii) Suppose $\mu_{1}=\mu_{2}=\cdots=\mu_{k}=\lambda_{1}$, and $\mu_{k+1}=\cdots=$ $\mu_{n}=\lambda_{2}$, for $1 \leq k \leq n$.

If $b=-1$, we get $\lambda_{1} \lambda_{2}=1$ and $M_{h, 5, k, 1 / \sqrt{\lambda_{1}^{2}-1}}=\{x \in$ $\left.\mathbb{U}^{n+1} ;\left(x^{1}\right)^{2}+\cdots+\left(x^{k+1}\right)^{2}=1 /\left(\lambda_{1}^{2}-1\right)\right\}$, with $A=\lambda_{1} I_{k} \oplus$ $\left(1 / \lambda_{1}\right) I_{n-k}$

In the sequel, let $M$ be a hypersurface of $\mathbb{S}^{n+1}$ which is isoparametric of type $l$; that is, the constant principal curvatures of $M$ are $\lambda_{1}>\cdots>\lambda_{l}$. It is known (see $[12,13]$ ) that $l \in\{1,2,3,4,6\}$ and for $l \leq 2$ we obtain the following.

(i) For $l=1, M$ is umbilical: $M_{\mathrm{sph}, r}=\left\{x \in \mathbb{S}^{n+1} ; x^{n+2}=\right.$ $\left.\sqrt{1-r^{2}}\right\}$ for $r \in(0,1)$ with $A=\left(\sqrt{1-r^{2}} / r\right) I . M_{\mathrm{sph}, r}$ is isometric to $S^{n}(r)$.

(ii) For $l=2, M$ is the generalized Clifford torus $M_{\mathrm{sph}, r_{1}, r_{2}}=$ $S^{m}\left(r_{1}\right) \times S^{n-m}\left(r_{2}\right)$ with $r_{1}^{2}+r_{2}^{2}=1$ and $1 \leq m<n$. From $\left[14\right.$, p. 86] we have $r_{1}=1 / \sqrt{1+\lambda_{1}^{2}}$ and $r_{2}=1 / \sqrt{1+\lambda_{2}^{2}}$ with $\lambda_{1} \lambda_{2}=-1$. 
Theorem 6. The only generalized golden-shaped hypersurfaces of $\mathbb{S}^{n+1}$ are as follows.

Case $1\left(\right.$ If $\left.a^{2}+4 b=0\right)$. Consider the following:

$$
M_{s p h, 2 / \sqrt{a^{2}+4}}=\left\{x \in \mathbb{S}^{n+1} ; x^{n+2}=\frac{|a|}{\sqrt{a^{2}+4}}\right\}
$$

which is isometric to $S^{n}\left(\left(a^{2}+4\right) / 4\right)$.

Case $2\left(\right.$ If $\left.a^{2}+4 b>0\right)$.

(i) For $l=1$,

$$
M_{s p h, 1 / \sqrt{\lambda_{1}^{2}+1}}=\left\{x \in \mathbb{S}^{n+1} ; x^{n+2}=\frac{\left|\lambda_{1}\right|}{\sqrt{\lambda_{1}^{2}+1}}\right\}
$$

which is isometric to $S^{n}\left(\lambda_{1}^{2}+1\right)$;

$$
M_{s p h, 1 / \sqrt{\lambda_{2}^{2}+1}}=\left\{x \in \mathbb{S}^{n+1} ; x^{n+2}=\frac{\left|\lambda_{2}\right|}{\sqrt{\lambda_{2}^{2}+1}}\right\}
$$

which is isometric to $S^{n}\left(\lambda_{2}^{2}+1\right)$.

(ii) If $b=1$ and for $l=2$,

$$
M_{s p h, r_{1}, r_{2}}=S^{m}\left(r_{1}\right) \times S^{n-m}\left(r_{2}\right)
$$

with $r_{1}^{2}+r_{2}^{2}=1$ and for $1 \leq m<n$.

Proof.

Case 1. Suppose $a^{2}+4 b=0, \lambda_{1}=\lambda_{2}=a / 2$; we get $\mu_{1}=$ $\mu_{2}=\cdots=\mu_{n}=a / 2$ and $c=a^{2} / 4+1>0$. Then $M_{\mathrm{sph}, 2 / \sqrt{a^{2}+4}}=$ $\left\{x \in \mathbb{S}^{n+1} ; x^{n+2}=|a| / \sqrt{a^{2}+4}\right\}$ which is isometric to $S^{n}\left(\left(a^{2}+\right.\right.$ 4)/4).

Case 2. Suppose $a^{2}+4 b>0$; we distinguish the following two cases:

(i) If $\mu_{1}=\mu_{2}=\cdots=\mu_{n}=\lambda_{1}$, we get $c=\lambda_{1}^{2}+1>0$ and $M_{\text {sph }, 1 / \sqrt{\lambda_{1}^{2}+1}}=\left\{x \in \mathbb{S}^{n+1} ; x^{n+2}=\left|\lambda_{1}\right| / \sqrt{\lambda_{1}^{2}+1}\right\}$ which is isometric to $S^{n}\left(\lambda_{1}^{2}+1\right)$.

If $\mu_{1}=\mu_{2}=\cdots=\mu_{n}=\lambda_{2}$, we get $c=\lambda_{2}^{2}+1>0$ and $M_{\text {sph }, 1 / \sqrt{\lambda_{2}^{2}+1}}=\left\{x \in \mathbb{S}^{n+1} ; x^{n+2}=\left|\lambda_{2}\right| / \sqrt{\lambda_{2}^{2}+1}\right\}$ which is isometric to $S^{n}\left(\lambda_{2}^{2}+1\right)$.

(ii) If $b=1$ and, for $l=2$, we get $\mu_{1}=\cdots=\mu_{m}=\lambda_{1}$ and $\mu_{m+1}=\cdots=\mu_{n}=\lambda_{2}$, for $1 \leq m<n, c_{1}=\lambda_{1}^{2}+1>0$, $c_{2}=\lambda_{2}^{2}+1>0$, and $\lambda_{1} \lambda_{2}=1$. Consider

$$
r_{1}^{2}+r_{2}^{2}=\frac{1}{c_{1}}+\frac{1}{c_{2}}=\frac{1}{\lambda_{1}^{2}+1}+\frac{1}{\lambda_{2}^{2}+1}=1 .
$$

Then $M_{\mathrm{sph}, r_{1}, r_{2}}=S^{m}\left(r_{1}\right) \times S^{n-m}\left(r_{2}\right)$ with $r_{1}^{2}+r_{2}^{2}=1$.

Remark 7. Theorems 4, 5, and 6 are the generalization of the results in [6]. That is, when $a=1$ and $b=1$, these three theorems are the same as theorems in [6].

\section{Generalized Product-Shaped Hypersurfaces}

In [4], it is proved that there exists a natural relationship between golden structures and almost product structures and this naturally leads to the following definition.

Definition 8. $M$ is called product-shaped hypersurface of $M^{n+1}(c)$ if $A^{2}=I$.

In this paper, we give the definition of generalized product-shaped hypersurface of $M^{n+1}(c)$.

Definition 9. $M$ is called generalized product-shaped hypersurface of $M^{n+1}(c)$ if $A^{2}=b I$, where $I$ is the identity on the tangent bundle of $M$ and $b$ is a constant satisfying $b \geq 0$.

It follows that the principal curvatures of $M$, as eigenvalues of $A$, are the golden mean $\lambda_{1}=\sqrt{b}$ and $\lambda_{2}=-\sqrt{b}$ if $b>0$. The principal curvatures of $M$, as eigenvalues of $A$, especially, are the golden mean $\lambda_{1}=\lambda_{2}=0$ if $b=0$. With similar computations as in Section 2, we obtain the following theorem.

Theorem 10. The product-shaped hypersurfaces are as follows: (1) For $\mathbb{R}^{n+1}$

(i) if $b=0$,

$$
M=\left\{x \mid x^{n+1}=0\right\}
$$

which is totally geodesic and is isometric to $\mathbb{R}^{n}$. In this case, the second fundamental form $A$ is zero;

(ii) if $b>0$,

$$
M=S^{n}\left(\frac{1}{\sqrt{b}}\right)
$$

(2) For $\mathbb{\boxplus}^{n+1}$

(i) if $b=0$, for $1 \leq k \leq n+1$,

$$
M_{h, 1, k}=\left\{x \in \mathbb{H}^{n+1} ; x^{k}=0\right\} ;
$$

in this case $A=O ; M$ is totally geodesic and is in fact just $\mathbb{Q}^{n}$;

(ii) if $0<b<1$,

$$
M_{h, 2, k, 1 / \sqrt{1-b}}=\left\{x \in \mathbb{\boxplus}^{n+1} ; x^{k}=\frac{1}{\sqrt{1-b}}\right\}
$$

for $1 \leq k \leq n+1$ which is isometric to $\mathbb{\boxplus}^{n}(b-1)$;

(iii) if $b=1$,

$$
M_{h, 3}=\left\{x \in \mathbb{H}^{n+1} ; x^{n+2}=x^{n+1}+1\right\}
$$

which is isometric to $\mathbb{R}^{n}$; 
(iv) if $b>1$,

$$
\begin{aligned}
& M_{h, 4,1 / \sqrt{b-1}} \\
& \quad=\left\{x \in \mathbb{H}^{n+1} ;\left(x^{1}\right)^{2}+\cdots+\left(x^{n+1}\right)^{2}=\frac{1}{b-1}\right\}
\end{aligned}
$$

which is isometric to $S^{n}(1 / \sqrt{b-1})$.

(3) For $\mathbb{S}^{n+1}$

(i) if $b=0$,

$$
M_{s p h, 1}=\left\{x \in \mathbb{S}^{n+1} ; x^{n+2}=0\right\}
$$

which is isometric to $S^{n}(1)$;

(ii) if $b>0$,

$$
M_{s p h, 1 / \sqrt{b+1}}=\left\{x \in \mathbb{S}^{n+1} ; x^{n+2}=\frac{\sqrt{b}}{\sqrt{b+1}}\right\}
$$

which is isometric to $S^{n}(b+1)$.

If $b=1$, especially, and, for $1 \leq m<n$,

$$
M_{s p h, 1 / \sqrt{2}, 1 / \sqrt{2}}=S^{m}\left(\frac{1}{\sqrt{2}}\right) \times S^{n-m}\left(\frac{1}{\sqrt{2}}\right) .
$$

\section{Competing Interests}

[7] C.-E. Hrețcanu and M. Crasmareanu, "Metallic structures on riemannian manifolds," Revista de la Union Matematica Argentina, vol. 54, no. 2, pp. 15-27, 2013.

[8] C. Özgür and N. Yilmaz Özgür, "Classification of metallic shaped hypersurfaces in real space forms," Turkish Journal of Mathematics, vol. 39, no. 5, pp. 784-794, 2015.

[9] G. Rotondaro, G. Sparano, and G. Vilasi, "Geometrical aspects of mixed torsionless tensor fields," International Journal of Geometric Methods in Modern Physics, vol. 3, no. 7, pp. 13811394, 2006.

[10] A. C. Coken, “On Euler's theorem in semi-Euclidean spaces $\mathbb{E}_{v}^{n+1}$, , International Journal of Geometric Methods in Modern Physics, vol. 8, no. 5, pp. 1117-1129, 2011.

[11] T. E. Cecil, "Isoparametric and Dupin hypersurfaces," Symmetry, Integrability and Geometry: Methods and Applications (SIGMA), vol. 4, article 062, 2008.

[12] P. J. Ryan, "Hypersurfaces with parallel Ricci tensor," Osaka Journal of Mathematics, vol. 8, pp. 251-259, 1971.

[13] P. J. Ryan, "Homogeneity and some curvature conditions for hypersurfaces," Tohoku Mathematical Journal, vol. 21, no. 3, pp. 363-388, 1969.

[14] M. Djoric and M. Okumura, CR Submanifolds of Complex Projective Space, vol. 19 of Developments in Mathematics, Springer, New York, NY, USA, 2010.

The authors declare that they have no competing interests.

\section{Acknowledgments}

This work is supported by NSFC (nos. 11371076 and 11431009).

\section{References}

[1] S. K. Sen and R. P. Agarwal, "Golden ratio in science, as random sequence source, its computation and beyond," Computers and Mathematics with Applications, vol. 56, no. 2, pp. 469-498, 2008.

[2] R. Heyrovska, "The Golden ratio, ionic and atomic radii and bond lengths," Molecular Physics, vol. 103, no. 6-8, pp. 877-882, 2005.

[3] L. Marek-Crnjac, "The golden mean in the topology of fourmanifolds, in conformal field theory, in the mathematical probability theory and in Cantorian space-time," Chaos, Solitons and Fractals, vol. 28, no. 5, pp. 1113-1118, 2006.

[4] M. Crasmareanu and C.-E. Hrețcanu, "Golden differential geometry," Chaos, Solitons and Fractals, vol. 38, no. 5, pp. 12291238, 2008.

[5] C.-E. Hretcanu and M.-C. Crasmareanu, "Applications of the golden ratio on Riemannian manifolds," Turkish Journal of Mathematics, vol. 33, no. 2, pp. 179-191, 2009.

[6] M. Crasmareanu, C.-E. Hrețcanu, and M.-I. Munteanu, "Golden- and product-shaped hypersurfaces in real space forms," International Journal of Geometric Methods in Modern Physics, vol. 10, no. 4, Article ID 1320006, 2013. 


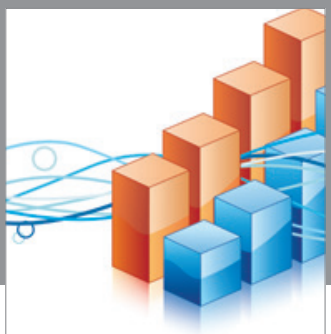

Advances in

Operations Research

vatem alat4

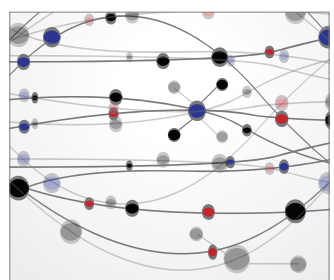

\section{The Scientific} World Journal
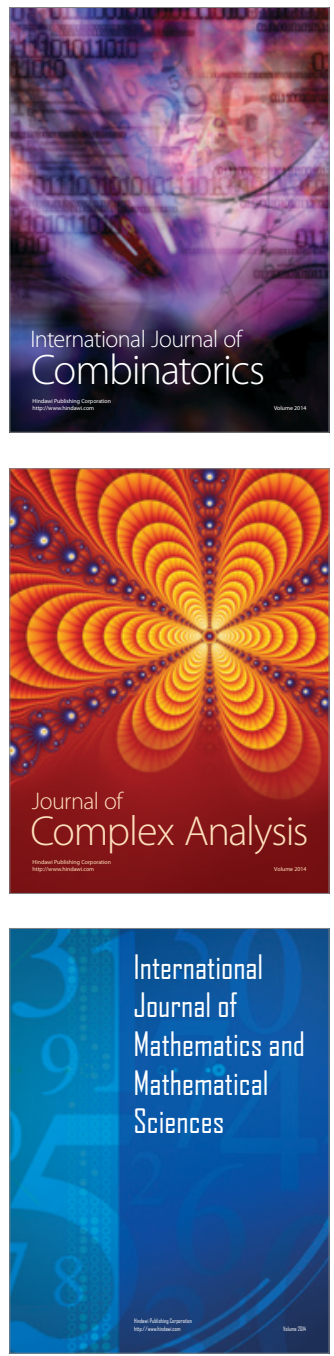
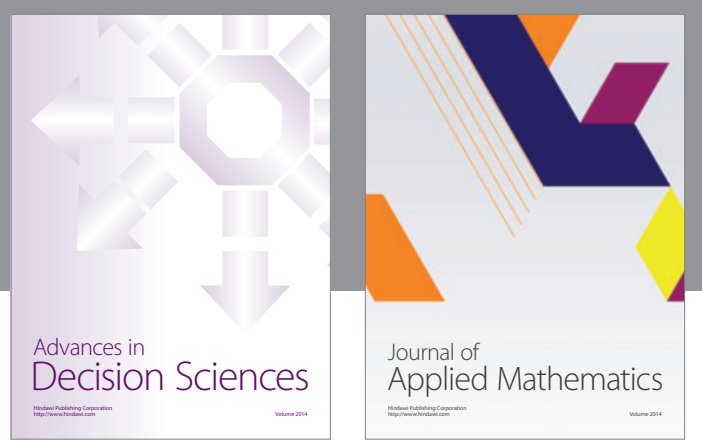

Algebra

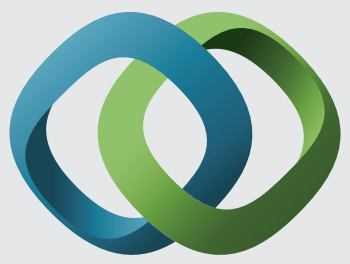

\section{Hindawi}

Submit your manuscripts at

http://www.hindawi.com
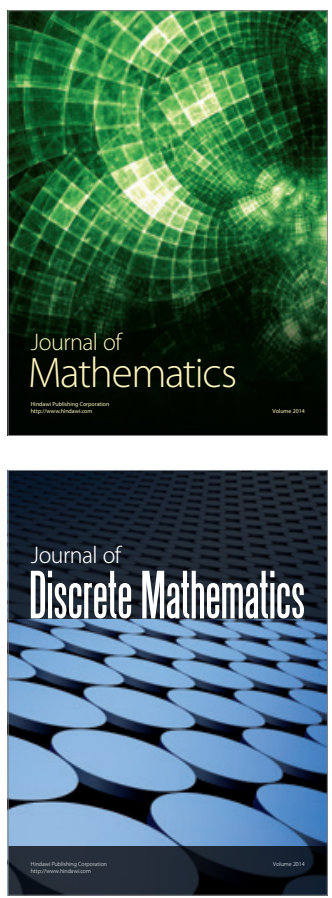

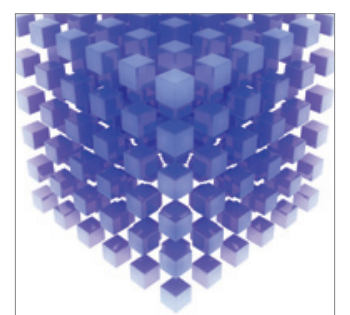

Mathematical Problems in Engineering
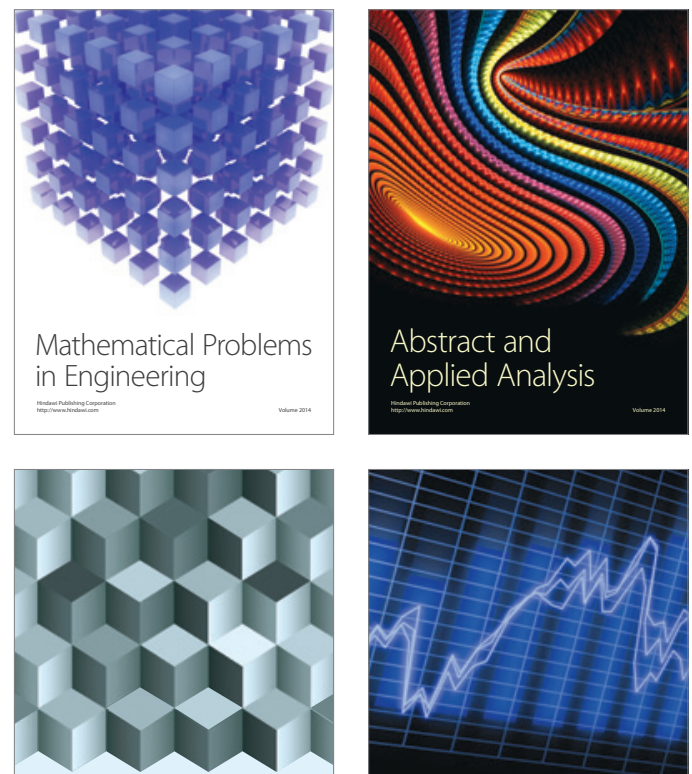

Journal of

Function Spaces

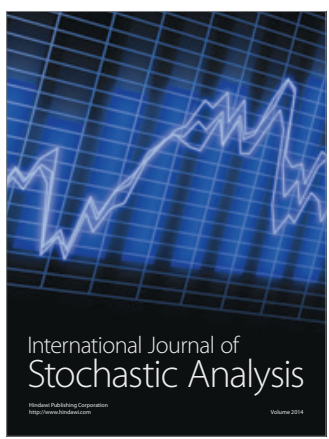

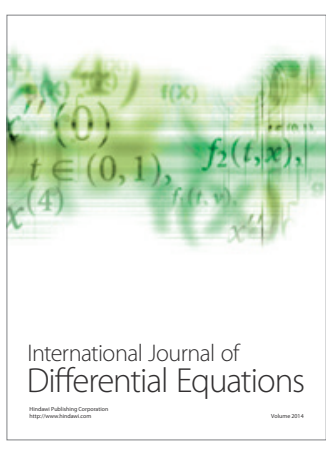
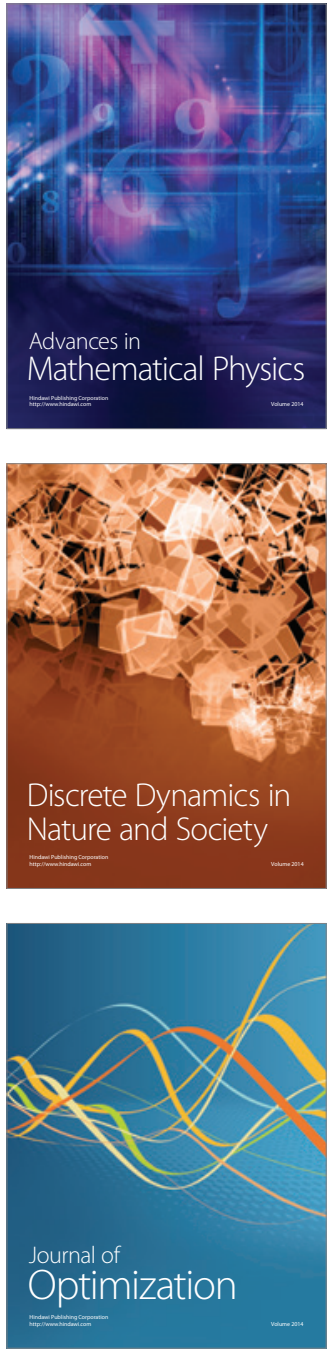